\title{
Cross-Visit Tumor Sub-segmentation and Registration with Outlier Rejection for Dynamic Contrast-Enhanced MRI Time Series Data
}

\author{
G.A. Buonaccorsi ${ }^{1,3}$, C.J. Rose ${ }^{1,3}$, J.P.B. O'Connor ${ }^{1,3}$, C. Roberts ${ }^{1,3}$, Y. Watson ${ }^{1,3}$, \\ A. Jackson ${ }^{1,3}$, G.C. Jayson ${ }^{2,3}$, and G.J.M. Parker ${ }^{1,3}$ \\ ${ }^{1}$ Imaging Science and Biomedical Engineering, School of Cancer and Imaging Sciences, \\ University of Manchester, Manchester, United Kingdom \\ ${ }^{2}$ CRUK Dept of Medical Oncology, Christie Hospital, Manchester, United Kingdom \\ ${ }^{3}$ The University of Manchester Biomedical Imaging Institute, University of Manchester, \\ Manchester, United Kingdom
}

\begin{abstract}
Clinical trials of anti-angiogenic and vascular-disrupting agents often use biomarkers derived from DCE-MRI, typically reporting whole-tumor summary statistics and so overlooking spatial parameter variations caused by tissue heterogeneity. We present a data-driven segmentation method comprising tracer-kinetic model-driven registration for motion correction, conversion from MR signal intensity to contrast agent concentration for cross-visit normalization, iterative principal components analysis for imputation of missing data and dimensionality reduction, and statistical outlier detection using the minimum covariance determinant to obtain a robust Mahalanobis distance. After applying these techniques we cluster in the principal components space using $k$-means. We present results from a clinical trial of a VEGF inhibitor, using time-series data selected because of problems due to motion and outlier time series. We obtained spatially-contiguous clusters that map to regions with distinct microvascular characteristics. This methodology has the potential to uncover localized effects in trials using DCE-MRI-based biomarkers.
\end{abstract}

Keywords: DCE-MRI, PCA, $k$-means, tracer kinetic modeling, image registration, imputation, outlier detection, minimum covariance determinant.

\section{Introduction}

Biomarkers derived from quantitative Dynamic Contrast-Enhanced MRI (DCE-MRI) data are used in clinical trials to support early decisions on the viability of emerging anti-angiogenic and vascular-disrupting agents [1]. A common approach is to acquire a time series of 3-D images at regular time intervals bracketing the injection of a contrast agent and then apply voxel-by-voxel analyses to generate 3-D parameter maps, e.g. using tracer-kinetic modeling [2]. Standard practice [1] is to report statistics for a volume of interest (VOI) that identifies the target tissues, e.g. a whole tumor. This practice discards the spatially-heterogeneous information in the parametric maps. Several recent studies have presented quantitative analyses that aim to describe this heterogeneous structure [3-5]. 
Our proposal is to partition tumors into 3-D sub-regions using a novel segmentation based on the DCE time series data. To obtain a consistent partitioning for multiple scan visits, we pool data from all visits for a given patient. Thus we may apply statistical analyses to tumor sub-regions to reveal localized variations in treatment response that would be masked in a whole-tumor VOI analysis. Our method requires no prior knowledge or assumptions about the form of the time series data.

A preliminary study [6] found that segmentation using dimensionality reduction by standard principal components analysis (PCA) and clustering by standard $k$-means was not feasible with motion-corrupted or missing data and was highly susceptible to outliers. In this study we present a procedure incorporating registration, missing value imputation and outlier detection and we demonstrate its robustness.

\section{Methods}

\subsection{Data Acquisition and Data Set Selection}

In a clinical trial of an angiogenesis inhibitor [7], patients had 6 MRI visits: 2 pretreatment scans, within 7 days prior to treatment; and 4 post-treatment scans ( 4 hours, 2, 8 and 12 days after treatment). All lesions were liver metastases arising from primary colorectal tumors. At each visit we acquired 3-D spoiled gradient echo (SPGR) images on a Philips $1.5 \mathrm{~T}$ Intera scanner for baseline $T_{1}$ estimation ( 3 images with flip angles of $2^{\circ}, 10^{\circ}$ and $30^{\circ}$ ) and for the dynamic time series ( 75 images: flip angle $20^{\circ}$, temporal resolution $4.97 \mathrm{~s}$, voxel matrix 128 x $128 \times 25$ ). Omniscan (Amersham Health, Amersham) was injected as a single bolus (dose $0.1 \mathrm{mmol} / \mathrm{kg}$ ) after the $5^{\text {th }}$ dynamic image, at a rate of $3 \mathrm{ml} / \mathrm{s}$ using a power injector (Spectris MR). To compensate for visit-to-visit variations in bolus arrival time we removed all time series images prior to the first appearance of contrast agent in the aorta in a central slice, giving a variable number $(T)$ of time points (range 65-68). A research radiographer manually defined 3-D tumor VOIs on co-localized $\mathrm{T}_{1}$-and $\mathrm{T}_{2}$-weighted images.

To test the segmentation, we selected 3 tumors in 3 patients with specific data characteristics: Patient 1 had significant motion corruption but few time series that were sufficiently different from the norm to be classed as outliers; Patient 2 had low motion but several outliers and Patient 3 had both motion and outliers.

\subsection{Motion Correction}

DCE-MRI presents difficulties for established registration methods, which readily deal with contrast variations but may fail when new image features arise due to contrast enhancement. We used tracer-kinetic model-driven registration (TKMDR) for the time-series images-for details of the registration procedure see [8]. Standard TKMDR does not correct motion of the pre-contrast variable flip-angle images relative to the time series, leaving the possibility of misregistration of $T_{l}(0)$ maps and leading to corruption of the cross-visit normalization (Section 2.3). We therefore additionally used a linear registration [9] to align each variable flip-angle image to the pre-contrast TKMDR synthetic target images. 


\subsection{Cross-Visit Normalization}

Raw DCE-MRI data from different patient visits are subject to unpredictable variations in scanner gain and potentially in native tumor relaxation time characteristics, so before pooling we normalized the time-series data to ensure that the scaling differences did not cause voxels from different visits to map to distinct clusters. Tracer kinetic models are typically expressed in terms of the tracer concentration $[C A](t)$ [2], which we used to normalize the data based on the physics of the data acquisition.

The conversion required the equation:

$$
[C A](t)=1 / r_{1}\left(1 / T_{1}(t)-1 / T_{1}(0)\right),
$$

where $r_{l}$ is the spin-lattice relaxivity constant, and $t$ is time. We used the standard SPGR equation [10] to estimate the pre-contrast longitudinal relaxation time, $T_{l}(0)$, from the variable-flip angle images and to derive $T_{l}(t)$ from the dynamic images.

\subsection{Dimensionality Reduction with Missing Value Imputation}

Each single-voxel $[C A](t)$ series is a vector in a $T$-dimensional data space $(T \geq 65)$, and it was desirable to reduce dimensionality prior to clustering. In addition, particularly in the presence of noise, low $T_{l}$ values may give rise to physiologically unfeasible $[C A](t)$ values from equation (1) - filtering to remove the most extreme values resulted in missing data for the corresponding vector elements. We employed a modified PCA to address both issues.

The $N x T$ data matrix for PCA pooled the time series data for each voxel of the tumor VOI for each visit for the given patient ( $N$ is the number of voxels in the pooled VOIs). Missing-value imputation was based on approximate reconstruction of the data via PCA, using the equation:

$$
\vec{y}=\sum_{t=1}^{r} w_{t} \vec{p}_{t}+\sum_{t=r+1}^{T} w_{t} \vec{p}_{t}=\hat{\vec{y}}+\vec{\varepsilon}
$$

where $\vec{y}$ is a $T$-dimensional time series data vector, the subscript $t$ is the time point index, $T$ is the number of time points, $\vec{p}_{t}$ is a $T$-dimensional principal component (PC) i.e. an eigenvector (obtained via singular value decomposition) of the sample covariance matrix for the data, $w_{t}$ is a scalar weighting factor (derived from the PCA), $\hat{\hat{y}}$ is the estimate of $\vec{y}$ obtained using $r$ PCs (see below) and $\vec{\varepsilon}$ is a $T$-dimensional residual vector. For each missing element of each $\vec{y}$, imputation required an initial estimate that was iteratively refined by replacement with $\hat{\vec{y}}$ until the sum of the elements of $\vec{\varepsilon}$ for all missing values became arbitrarily small [11]. Standard practice initializes using the data matrix row or column means [12], but as time-series data are correlated we initialized using the mean of the adjacent non-missing time points. To obtain a close approximation, we selected $r$ such that the PCA explained $97.5 \%$ of the variance. We then back-substituted the imputed values into the original time series in place of the missing values. We used the resulting PCs for dimensionality reduction, 
retaining the subset of $d$ principal components indicated by examination of logeigenvalue plots ( $d \neq r$ : $d$ ranged from 3 to 7 PCs and $r$ was typically around 40 PCs).

\subsection{Outlier Detection}

The filtering of erroneous [CA] values (Section 2.4) was extremely conservative, to avoid unintended distortion of the data. The remaining artefactually high [CA] values resulted in time series that deviated sufficiently from the norm to be considered outliers. These outliers may be detected in the multivariate space defined by the retained PCs by thresholding the Mahalanobis distance $(M)$ at a critical value $\left(M_{C}\right)$ obtained from the $\chi^{2}$ distribution with degrees of freedom $(d)$ equal to the number of PCs retained for dimensionality reduction at a chosen percentile $\alpha$ (typically 97.5\%) [13]:

$$
M=(p-\bar{p})^{\prime} \sum^{-1}(p-\bar{p}) ; \quad M_{C}=\operatorname{inv}\left(\sqrt{\chi_{d}^{2}(\alpha)}\right)
$$

where $\Sigma$ is the sample covariance matrix and the prime indicates matrix transposition. The vectors $p$ and $\bar{p}$ are the truncated $d$-dimensional PCs from Section 2.4.

If $\bar{p}$ and $\Sigma$ are estimated as the classical mean and sample covariance matrix then each will be strongly affected by the outliers we wish to detect (the masking problem [13]), so we employed a robust Mahalanobis distance using the minimum covariance determinant (MCD) estimators of $\bar{p}$ and $\Sigma$ [14], as obtained with the FAST-MCD algorithm from the Matlab Library for Robust Analysis [15] (we increased $\alpha$ to $99.99 \%$ to reduce the potential misclassification of valid data as outliers). For the purposes of this study, any voxel time series with $M_{\text {voxel }}>M_{C}$ was considered to be an outlier, and the entire $[C A](t)$ series was removed from further analysis.

\subsection{Clustering}

We performed 10 repetitions of multi-start $k$-means clustering [16] in the data space of the retained principal components. Initial cluster centers were chosen randomlydifferent randomizations could converge to different solutions, so we retained the solution with the minimum sum of the squared Euclidean distance from each data point to its cluster mean vector. Clustering was done separately for each patient, but the data from all visits for the same patient were pooled. We set $k=7$ to reflect typical tumor structure in a DCE-MRI data set: voxels could correspond to tumor enhancing rim, tumor non-enhancing core or surrounding liver and to partial voluming among these tissue types. Alternative arrangements are clearly possible. Only the retained principal components of the $[C A](t)$ series data were used in the clustering procedure-no spatial regularization or other spatial (e.g. neighborhood or connectivity) information was used, and clusters were therefore not constrained to be spatiallycontiguous. For visualization purposes, we gave each cluster an integer label.

\section{Results}

Figure 1 shows sample imputation results for two voxel time series, each of which had 3 missing values. The imputed values generally lie within the range of the time series data, and do not significantly alter its observed pattern. 

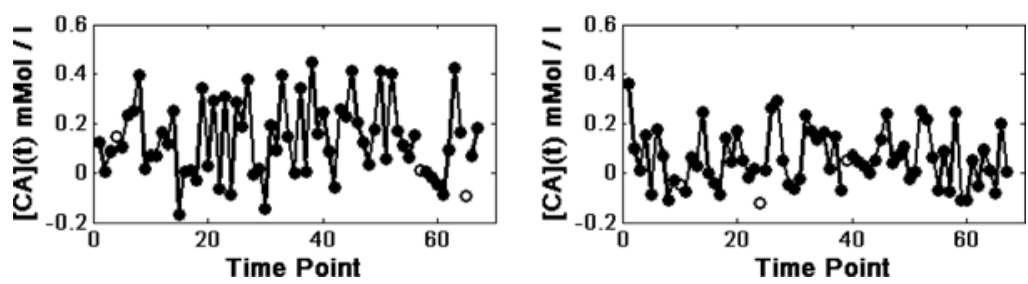

Fig. 1. Contrast agent concentration time series before (line with solid circles) and after (open circles) imputation of missing data for two voxel time series (Patient 3, post-registration)

(a)

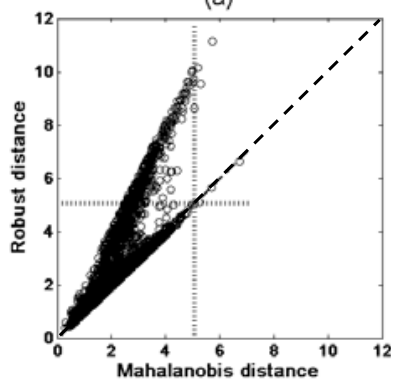

(b)

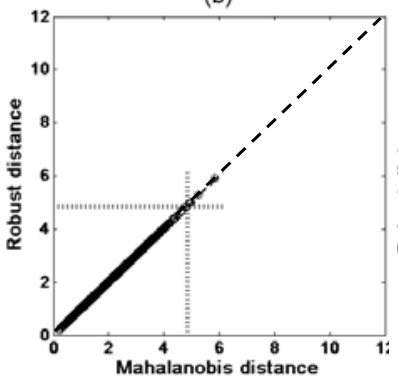

(c)

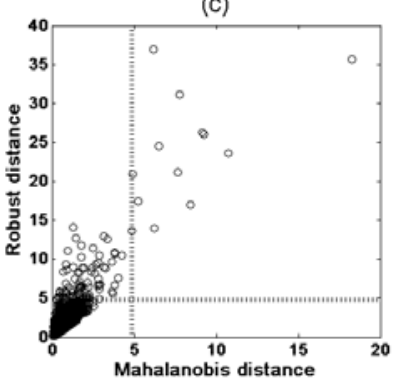

Fig. 2. Distance-Distance scatterplots of robust versus classical Mahalanobis distance: (a) preand (b) post-registration for Patient 1; (c) post-registration for Patient 3. Hatched lines show $M_{C}$ values calculated using Equation (3); dashed lines in (a) and (b) show the line of equality. The data have been pooled for all visits.

The pre-registration Distance-Distance plot for Patient 1 (Figure 2a) shows two data sub-populations. The post-registration plot (Figure $2 b$ ) indicates that these were due to motion, which has been adequately corrected. The close correspondence of robust and classical Mahalanobis distance also indicates that the Patient 1 data had very few outliers. Figure 2c illustrates that outliers persist after registration for Patient 3. The Distance-Distance plot for Patient 2 was similar to Figure $2 \mathrm{c}$ and is not shown.

Figure 3 illustrates the effect of TKMDR on the segmentation results. While the differences are relatively subtle, the post-registration cluster images are visibly less fragmented in nature (though the effect is subtle). After treatment, the typical enhancing-rim pattern of tumors is evident in that the blue and blue-green clusters correspond to low contrast agent uptake (clusters 1 and 7 in Figure 4d) while the red and orange clusters correspond to higher uptake (clusters 3 and 5 in Figure 4d). This is consistent with a post-treatment reduction in angiogenesis and/or microvessel density.

Table 1 provides further evidence of the presence of outliers in the very small clusters among the "Raw" data for Patient $2(\leq 0.15 \%$ of voxels) and Patient $3(\leq 0.35 \%$ of voxels). The very small clusters for Patient 2 had physiologically unfeasible mean $[C A](t)$ series (cf Figure $4 \mathrm{a}$ and $4 \mathrm{~b}$ ). Outlier removal eliminated the very small clusters (Table 1 "Patient 2: MCD") and returned all cluster mean $[C A](t)$ series to fall within the physiological range (Figure 4c). For Patient 2, the clusters after registration and 


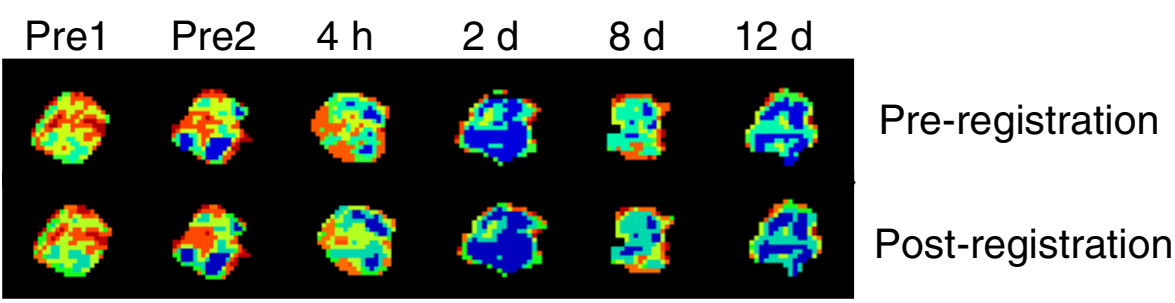

Fig. 3. Segmentation maps for clustering after outlier removal for Patient 1. Clusters are individually colour-coded using integer labels.

Table 1. Number of voxels per cluster for each patient before and after outlier removal using the robust MCD-based Mahalanobis distance. The data have been pooled for all visits.

\begin{tabular}{rcccccc} 
& \multicolumn{2}{c}{ Patient 1 } & \multicolumn{2}{c}{ Patient 2 } & \multicolumn{2}{c}{ Patient 3 } \\
Cluster & Raw & MCD & Raw & MCD & Raw & MCD \\
1 & 661 & 664 & 12 & 6383 & 764 & 462 \\
2 & 654 & 757 & 7 & 6327 & 819 & 291 \\
3 & 603 & 649 & 12603 & 3533 & 346 & 123 \\
4 & 1073 & 471 & 23283 & 1251 & 8 & 260 \\
5 & 758 & 619 & 1 & 6582 & 2 & 85 \\
6 & 494 & 1015 & 56 & 4426 & 1 & 509 \\
7 & 1054 & 1116 & 1 & 5300 & 344 & 452
\end{tabular}

outlier removal were stratified in mean $[C A](t)$ (Figure 4c), but for Patient 1 the clustering resulted in different mean $[C A](t)$ curve shapes (compare solid lines with dashed lines in Figure 4d), reflecting more complex enhancement characteristics.

\section{Discussion}

The methodology of this paper used TKMDR for motion correction, conversion of MR signal to $[C A](t)$ for cross-visit normalization, iterative PCA for missing data imputation and dimensionality reduction, and a robust Mahalanobis distance via MCD for outlier detection. After applying these techniques to DCE-MRI $[C A](t)$ series data, tumor sub-segmentation could be performed using standard $k$-means clustering.

The iterative PCA imputation provided reasonable $[C A](t)$ estimates (Figure 1), although one imputed value in each example fell lower than its neighbors - this may reflect the true data structure but could indicate a need to fine-tune the procedure by retaining more PCs, or increasing the number of iterations.

As the clustering procedure did not incorporate spatial regularization or any other spatial information, it is encouraging that the segmentation results showed a high level of spatial contiguity, in that the voxels of any given cluster were generally found grouped in the same spatial location (Figure 3) rather than being loosely scattered through the image volumes. 
(a)

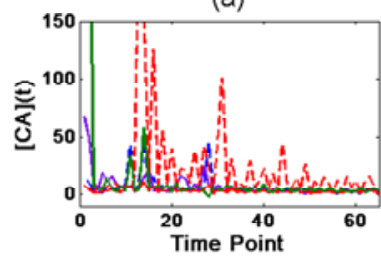

(b)

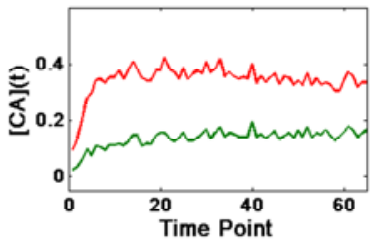

(c)

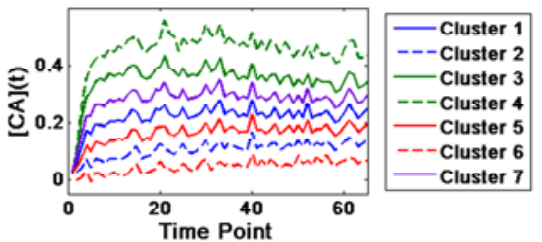

(d)

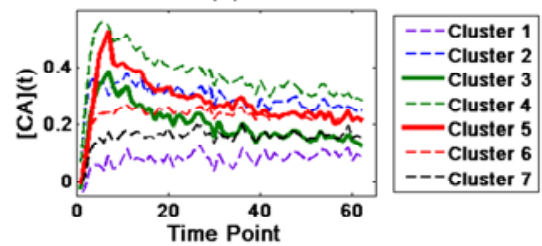

Fig. 4. Cluster mean $[C A](t)$ series for Patient 2 before $(\mathrm{a}, \mathrm{b})$ and after (c) outlier removal, and for Patient 1 after outlier removal (d). Note the scale change in (a), for which all clusters have [CA] values that exceed the maximum of c. $20 \mathrm{mmol} / \mathrm{l}$ observed in the aorta. The data have been pooled for all visits.

The clustering results can be skewed by even a small number of outliers if they lie far enough from the bulk of the data, as illustrated by the unphysiological mean $[C A](t)$ series for the very small clusters for Patient 2 (Table 1 and Figure 4a). Identification and removal of the outliers restored the mean $[C A](t)$ series to the physiological range (Figures $4 \mathrm{c}$ and $4 \mathrm{~d}$ ).

In the absence of outliers, Distance-Distance plots take the form of Figure $2 b$ because the MCD and classical estimates for the data centroid and covariance matrix match, as do the corresponding Mahalanobis distances. Outliers pull the classical estimates towards themselves [13], so their robust Mahalanobis distances are greater than the classical ones, as in Figure 2a and 2c. Registration could reduce the number of outlier time series (Figure $2 \mathrm{a}$ and $2 \mathrm{~b}$ ) but not in all cases (Figure 2c) and its effect on cluster structure was subtle (Figure 3). Note that our additional registration of the variable flip-angle images to the pre-contrast TKMDR synthetic target images (Section 2.2) ensured that $T_{l}(0)$ and $T_{l}(t)$ in Equation (1) arose from the same voxels when applying cross-visit normalization.

Outliers have been defined as data that are sufficiently different from the norm to suggest a different generative mechanism [12]. For this study we simply removed outliers to robustify the clustering, but it would be informative to gather outliers into additional clusters, which could be examined to understand their origin. While some outliers will arise from artefactually low $T_{l}(t)$ values (Section 2.4), some may also provide insight into treatment effects. Finally, as ground truth is impossible to establish with patient data, simulation studies would provide further validation.

\section{Conclusions}

We have described a method for unsupervised, data-driven segmentation for DCEMRI time series data, applicable to multi-visit clinical trials. The method uses registration for motion-correction, imputation of missing data values and statistical outlier 
identification and rejection. The resulting clusters are in general spatially contiguous and have physiologically reasonable mean $[C A](t)$ series. Our procedure will allow statistical analyses to be applied to sub-regions of tumors to reveal localized variations in treatment response that would be masked in a whole-tumor VOI analysis. Such results could, for example, influence decisions on whether or not to proceed with the development of a particular drug.

Acknowledgments. This project was partially-funded by CRUK grants C237/A6295 and C19221/A6086.

\section{References}

1. O'Connor, J.P.B., Jackson, A., et al.: DCE-MRI biomarkers in the clinical evaluation of antiangiogenic and vascular disrupting agents. Br. J. Cancer 96, 189-195 (2007)

2. Jackson, A., Buckley, D.L., Parker, G.J.M. (eds.): Dynamic contrast-enhanced magnetic resonance imaging in oncology. Springer, Berlin (2005)

3. Rose, C.J., Mills, S., et al.: Quantifying spatial heterogeneity in Dynamic ContrastEnhanced MRI Parameter Maps. Magn. Reson. Med. 62, 488-499 (2009)

4. Berry, L.R., Barck, K.H., et al.: Quantification of viable tumor microvascular characteristics by multispectral analysis. Magn. Reson. Med. 60, 64-72 (2008)

5. Carano, R.A.D., Ross, A.L., et al.: Quantification of tumor tissue populations by multispectral analysis. Magn. Reson. Med. 51, 542-551 (2004)

6. Buonaccorsi, G.A., Roberts, C., et al.: Cross-visit tumor sub-segmentation reveal localised response to anti-angiogenic treatment in DCE-MRI data. Proc. Int. Soc. Magn. Reson. Med. 18, 4813 (2010)

7. O'Connor, J.P.B., Carano, R.A.D., et al.: Quantifying antivascular effects of monoclonal antibodies to vascular endothelial growth factor: insights from imaging. Clin. Cancer Res. 15, 6674-6682 (2009)

8. Buonaccorsi, G.A., O’Connor, J.P.B., et al.: Tracer kinetic model-driven registration for dynamic contrast-enhanced MRI time-series data. Magn. Reson. Med. 58, 1010-1019 (2007)

9. Jenkinson, M., Bannister, P., et al.: Improved optimisation for the robust and accurate linear registration and motion correction of brain images. Neuroimage 17, 825-841 (2002)

10. Haase, A.: Snapshot FLASH MRI: application to $T_{1}, T_{2}$ and chemical shift imaging. Magn. Reson. Med. 13, 77-89 (1990)

11. Stanimirova, I., Daszykowski, M., et al.: Dealing with missing values and outliers in principal component analysis. Talanta 72, 172-178 (2007)

12. Walczak, B., Massart, D.L.: Dealing with missing data: Part I. Chemometr. Intell. Lab. Syst. 58, 15-27 (2001)

13. Hawkins, D.: Identification of Ouliers. Chapman \& Hall, London (1980)

14. Rousseeuw, P.J., van Zomeren, B.C.: Unmasking multivariate outliers and leverage points. J. Am. Stat. Assoc. 85, 633-659 (1990)

15. Verboven, S., Hubert, M.: LIBRA: a Matlab library for robust analysis. Chemometr. Intell. Lab. Syst. 75, 127-136 (2005)

16. Everitt, B.S.: Cluster Analysis. Edward Arnold, London (1993) 